\title{
Diagnostic Imaging in Neuroendocrine Tumors
}

H

ealth is priceless, but health care has a budget. In the era when the dream of personalized medicine may become a reality, there are not enough resources to allow the best diagnostic or therapeutic strategy in every situation. An individual is by definition different from all others. Because medicine is not an exact science that can always predict the best imaging modality for evaluation of patients, medical decisions are made on a probabilistic evaluation.

The value of a procedure should be estimated on the basis of parameters such as risk stratification, diagnostic accuracy, prognostic value, impact on management, duration of the diagnostic evaluation, and treatment. Therefore, the cost has to be estimated for the entire diagnostic and therapeutic course, not only for the single diagnostic procedure. With the increasing cost of health care, decisions should be based on a careful cost-effectiveness evaluation to achieve the optimal outcome at the lowest cost.

This general premise concurs with the results of a study by Etchebehere et al. (1) reported in this issue of The Journal of Nuclear Medicine. Their prospective trial compared lesion detectability between ${ }^{68} \mathrm{Ga}$-DOTATATE PET/CT, ${ }^{99 \mathrm{~m} T c-h y d r a z i n o n i c o t i n a m i d e}$

\section{See page 1598}

(HYNIC)-octreotide (somatostatin receptor scintigraphy [SSRS]) SPECT/CT, and whole-body diffusion-weighted MR imaging (WB DWI) in 19 consecutive patients with neuroendocrine tumors (NET). The authors concluded that, although their study was limited by the low number of observations, " ${ }^{68} \mathrm{Ga}$ PET/CT seems to be more sensitive for detection of well-differentiated NET lesions, especially for bone and unknown primary lesions. NET can be staged with ${ }^{68} \mathrm{Ga}$-DOTATATE PET/CT. WB DWI is an efficient new method with high accuracy and without ionizing radiation exposure. SSRS SPECT/CT should be used only when ${ }^{68} \mathrm{Ga}-$ DOTATATE PET/CT and WB DWI are not available."

This strategy has to be adapted to the procedures available locally and has to take into account local expertise and equipment.

PET/CT with DOTA peptides has a primary role in the staging of well-differentiated gastroenteropancreatic tumors. Its use is justified in NET patients for applications ranging from initial diagnosis to evaluation of tumor response. From a cost-effectiveness perspective, the use of new imaging procedures has to be integrated into the overall cost of patient care.

Received Sep. 2, 2014; revision accepted Sep. 4, 2014.

For correspondence or reprints contact: Luigi Mansi, Medicina Nucleare, Seconda Università di Napoli, Piazza Miraglia, 80138 Naples, Italy.

E-mail: luigi.mansi@unina2.it

Published online Sep. 18, 2014.

COPYRIGHT (C) 2014 by the Society of Nuclear Medicine and Molecular Imaging, Inc.

DOI: $10.2967 /$ jnumed.114.147082
Imaging procedures have a primary role to stage and restage patients with NET. Multislice triple-phase CT is usually the first procedure performed. When multislice CT is performed as a component of a hybrid system, its sensitivity may be increased when associated with DOTA peptide PET. Unknown small ileal lesions may be found with multislice-CT enterography.

MR imaging should be used on younger patients or for longterm surveillance and is also useful when multislice CT does not provide clear-cut results or contrast medium is contraindicated. MR imaging may provide better results than multislice CT for detection of lesions in the central nervous system and skeleton and to characterize lesions in the liver. For the latter indication, a similar role may be played by contrast-enhanced ultrasound, which also is useful for guiding biopsy. Endoscopic ultrasound may detect unknown small pancreatic lesions and provide guidance for biopsy. When MR imaging or SSRS SPECT (also without CT) is planned for restaging or for evaluation of tumor response, comparison with a previous study is needed.

Before therapy with cold or radiolabeled somatostatin analogs, evaluation with radionuclide diagnostic procedures is mandatory to assess the presence of somatostatin receptors and to evaluate renal toxicity before peptide receptor radionuclide therapy (PRRT). The best results are achieved when diagnostic and therapeutic radiotracers that have similar pharmacokinetics are used. ${ }^{111} \mathrm{In}$ pentetreotide (OctreoScan; Mallinckrodt Pharmaceuticals) may continue to play a clinical role before PRRT with either ${ }^{90} \mathrm{Y}$ - or ${ }^{177} \mathrm{Lu}-$ DOTA peptides because ${ }^{111}$ In has a longer half-life than ${ }^{68} \mathrm{Ga}$ and would allow individual dosimetric estimates.

${ }^{18}$ F-FDG PET has limited usefulness for staging because of its low sensitivity in well-differentiated NETs. Conversely, ${ }^{18} \mathrm{~F}-\mathrm{FDG}$ can be used for prognostic stratification of high-grade (G3) and selected G2 gastroenteropancreatic tumors, in which dedifferentiation may indicate the escape phenomenon-that is, the disappearance of somastatin receptors on neoplastic cells-defining the worst prognosis.

Several DOTA peptides radiolabeled with ${ }^{68} \mathrm{Ga}$, such as DOTATATE, DOTATOC, and DOTANOC, are available for clinical use. The distribution of somatostatin analogs is conditioned by their affinity to the 5 subtypes of somatostatin receptor, with diagnostic accuracy in NETs being due mainly to overexpression of somatostatin receptors 2 and 5 on neoplastic cells. Although rare possible mismatches are observable between diverse DOTA peptides, the data have not yet been able to determine the best radiotracer for individual patients. Similarly, no advantages have been observed from the use of DOTA-lanreotide, which shows different pharmacokinetics. PRRT should be preceded by a diagnostic evaluation, preferably using the same pharmaceutical.

There is great interest in the emergence of the so-called panreceptor somatostatin analogs, such as SOM230 (pasireotide), which can target a wider range of NET cells, including those not expressing somatostatin receptors 2 and 5. The availability of these analogs would be particularly important for identifying insulinoma, which is rarely detectable because of the low expression of somatostatin receptor 2 . 
L-3,4-dihydroxy-6- ${ }^{18} \mathrm{~F}$-fluorophenylalanine $\left({ }^{18} \mathrm{~F}-\mathrm{DOPA}\right)$ is a tracer for catecholamine metabolism and exhibits an intense concentration in NETs, independent of somatostatin receptor. Although the behavior of ${ }^{18} \mathrm{~F}$-DOPA and DOTA peptides is similar, with both being taken up by well-differentiated lesions, a mismatch between the two is sometimes observed. In clinical practice, DOTA peptides are generally preferred, having the additional advantages of wider availability and lower cost, and the indication for ${ }^{18} \mathrm{~F}-\mathrm{DOPA}$ may actually be mainly for patients in whom insulinoma is suspected and patients with paraganglioma (2). In patients with insulinoma, the use of glucagonlike peptide 1 receptor imaging, not yet in clinical practice, has also been proposed. Although there is no indication for ${ }^{18} \mathrm{~F}$-DOPA before PRRT, further studies are needed to evaluate its possible role in identifying patients who would benefit from ${ }^{131}$ I-metaiodobenzylguanidine therapy, which has a similar uptake mechanism.

Although radiolabeled somatostatin analogs have demonstrated a potential role to identify patients who would benefit from PRRT, clinical usefulness has not yet been found for radiolabeled neuropeptides targeting other receptors highly expressed in NETs, such as gastrin-releasing peptide, cholecystokinin $2, \alpha_{\nu} \beta_{3}$ integrin, neurokinin 1 , and glucagonlike peptide 1 .

In a comparison of procedures characterizing somatostatin receptors, DOTA peptide PET/CT is more accurate than SPECT/CT, which is more accurate than stand-alone SPECT, which is more accurate than planar imaging (1). Although having lower diffusion and a lack of comparative data, ${ }^{99 \mathrm{~m}} \mathrm{Tc}$-radiopeptides such as ${ }^{99 \mathrm{~m}} \mathrm{Tc}-$ HYNIC-octreotide seem superior to OctreoScan in diagnostic accuracy and cost-effectiveness, as well as being more available and having faster scanning times. OctreoScan remains a valuable alternative except for the detection of small primary lesions.

The higher sensitivity of DOTA peptide PET/CT than of SRRS SPECT for detection of lesions does not always affect the management of individual patients. Therefore, OctreoScan may continue to be the standard of care for determining the presence of disease and monitoring active lesions.

A unique indication for OctreoScan is radioguided surgery of concentrating tumors, including those, such as gastroenteropancreatic tumors and carcinoids, in which somatostatin receptor is highly expressed, and those, such as non-small cell lung carcinoma, in which the tracer is taken up by reactive cells surrounding a neoplasm not expressing somatostatin receptor $(3,4)$. OctreoScan could also be used to differentiate non-small cell lung carcinoma from small cell lung carcinoma. A study in which OctreoScan SPECT was performed at 4 and $24 \mathrm{~h}$ showed that although a stationary or increasing tumorto-background ratio was observed in small cell lung carcinoma (and carcinoids) on the earlier scan, a decreasing ratio was present in nonsmall cell lung carcinoma on the later scan (3). In small cell lung carcinoma the uptake is at the level of stationary neoplastic cells, and in non-small cell lung carcinoma the uptake is at least partly due to mobile cells, such as activated lymphocytes and macrophages.

Because of the high concentration of activated lymphocytes or macrophages in many inflammatory diseases, OctreoScan may be used not only to define the disease state but also to predict the effect of a therapeutic strategy using cold somatostatin analogs (or antiinflammatory drugs). For this reason, important clinical indications may exist in many benign diseases such as Graves exophthalmos, sarcoidosis, rheumatoid arthritis, inflammatory bowel disease, histiocytosis, and idiopathic pulmonary fibrosis. Investigation of the use of PET/CT with DOTA peptides or ${ }^{99 \mathrm{~m}} \mathrm{Tc}-\mathrm{HYNIC}$ octreotide for these indications needs to be strongly encouraged.

\section{DISCLOSURE}

No potential conflict of interest relevant to this article was reported.

Luigi Mansi

Vincenzo Cuccurullo

Seconda Università di Napoli

Naples, Italy

\section{REFERENCES}

1. Etchebehere E, de Oliveira Santos A, Gumz B, et al. ${ }^{68} \mathrm{Ga}$-DOTATATE PET/CT, ${ }^{99 \mathrm{~m}} \mathrm{Tc}-\mathrm{HYNIC}$-octreotide SPECT/CT, and whole-body MR imaging in detection of neuroendocrine tumors: a prospective trial. J Nucl Med. 2014;55:1598-1604.

2. Cuccurullo V, Mansi L. Toward tailored medicine (and beyond): the phaeochromocytoma and paraganglioma model. Eur J Nucl Med Mol Imaging. 2012;39:1262-1265.

3. Cascini GL, Cuccurullo V, Mansi L. The non tumour uptake of ${ }^{111}$ In-octreotide creates new clinical indications in benign diseases, but also in oncology. $\mathrm{QJ} \mathrm{Nucl}$ Med Mol Imaging. 2010;54:24-36.

4. Cascini GL, Cuccurullo V, Tamburrini O, Rotondo A, Mansi L. Peptide imaging with somatostatin analogues: more than cancer probes. Curr Radiopharm. 2013;6: $36-40$. 